\title{
Multi-period Sequential Synthesis of Heat Exchanger Networks and Utility Systems including storages
}

\author{
Alberto Mian $^{\mathrm{a}^{*}}$, Emanuele Martelli $^{\mathrm{b}}$ and Francois Marchal ${ }^{\mathrm{a}}$ \\ a Industrial Process Energy Systems Engineering (IPESE), Ecole Polytechnique Fdrale de Lau- \\ sanne, 1015, Lausanne, $\mathrm{CH}$ \\ ${ }^{\mathrm{b}}$ Politecnico di Milano, Dipartimento di Energia, Via Lambruschini 4, Milano, IT \\ *alberto.mian@epfl.ch
}

\begin{abstract}
This work proposes a sequential approach for the multi-period synthesis of Heat Exchanger Networks (HEN) and Utility Systems of chemical processes and energy systems, including thermal, electric and material storage. The optimization approach is sequential and it consists in three steps: (1) the multi-period Mixed Integer Linear Programming (MILP) energy integration model of Marechal and Kalitventzeff (2003) determines the optimal utility selection, size and operation scheduling (on/off) as well as the size of the storage system which minimize the linearized utility total costs for given Heat Recovery Approach Temperature (HRAT), (2) a modified version of the multi-period MILP minimum number of units problem Floudas and Grossmann (1986) determines the number of matches (heat exchanger units) between hot and cold streams such that the sum of the associated penalty levels are minimized, (3) the Non Linear Programming (NLP) multi-period HEN synthesis model proposed by Floudas and Grossmann (1987) finds the HEN with minimum area. In order to partially overcome limitations of the sequential approach, HRATs for each stream and for each time period, as well as penalty levels associated to each possible exchange and the sizes of available utilities are optimized using the derivative-free hybrid algorithm PGS-COM Martelli and Amaldi (2014).
\end{abstract}

Keywords: Thermal Storage, Heat Exchanger Network Synthesis, Multi-period Synthesis, Sequential approach

\section{Introduction}

The synthesis and design of multi-period heat exchanger networks and utility systems is a challenging and well explored field of research. In the context of multi-period problems, batch processes and in general energy and chemical plants, a key task is represented by the change in temperature and heat load of not only process streams but also utility streams when the part-load of the utilities is accounted. The integration of thermal storages represent a key aspect in the design of energy systems and chemical plants. By including thermal storage it is in fact possible to perform process-process heat recovery across time periods, and eventually reduce utility consumption, as well as utility size. As for the case of utility systems and heat exchanger network, the trade-offs between storage utility size, operating costs and investment costs of the corresponding heat exchanger network need to be accounted for. Cosidering the mathematical programming approach, the first formulation for scheduling storage operations in batch processes was proposed in Grossmann and Santibanez (1980). Recently Christidis et al. (2012) have addressed the problem of storage integration in combined heat and power plants. To the best of our knowledge, only one literature work couples storage utility design with heat exchanger network design; it is the work of 
Chen and Chou Chen and Ciou (2008), where the simultaneous multi-period synthesis approach for HEN is adopted. In this work we propose a novel sequential approach for the combined synthesis and optimization of utility systems and HEN with storage systems. In order to overcome the limitations of the sequential decomposition, HRATs of each streams, penalty levels of the matches, utility size and storage design variables are optimized by a global search derivative-free algorithm.

\section{Problem Statement}

The multi-period HEN and utility synthesis problem tackled in this work can be stated as follows: Given:

- a set of hot and cold process streams with given period dependent mass flow rates, period dependent inlet and outlet temperatures;

- a set of available utility systems (e.g., cooling water, boiler, multiple-level steam networks operating at fixed temperature and pressures, refrigeration cycle, heat pump, etc.);

- a set of available thermal storages (with given density, heat capacity of the heat transfer fluid, and geometry );

- cost data relative to heat exchangers, utility systems and thermal storages;

Determine:

- the optimal selection, size and load in each period of the utility systems;

- the optimal selection, size and load in each period of the storage utility systems;

- the optimum heat exchanger network configuration as well as design (inlet/outlet temperatures, heat load, area of each process-process, process-utility and utility-utility heat exchanger).

In order to develop an effective approach for tackling industrial scale problems, an improved version of the sequential approach originally proposed by Floudas and Grossmann (1987) has been revised. The multi-period utility integration and scheduling model proposed by Marechal and Kalitventzeff (2003) has been integrated with a revised version of the multi-period HEN optimization approach of Floudas and Grossmann (1987).

\subsection{Algorithm}

The first step consists in finding the utility load and scheduling for fixed size of utilities and for given operating reference loads. In particular, we have adapted the approach proposed by Marechal and Kalitventzeff (2003) in such a way to optimize the system scheduling for given utility sizes, for given Heat Recovery Approach Temperature (HRAT) of process and utilities. HRATs have to be specified for each stream and for each time period. In addition, in this formulation forbidden matches can be specified in order to account for engineering/safety constraints.

The second step consists in finding the number of connections between hot and cold process/process, process/utility and utility/utility streams, by considering process streams and utility loads previously determined (Step 1). In particular, the Multi-period Minimum Number of Unit model proposed by Floudas and Grossmann (1986) is modified by introducing Penalty Levels for each possible connection and by minimizing the total penalty associated to the activation of the connections. The use of PLs is intended to find solutions with improved compromise between number of units and heat exchanger area compared to the plain minimization of the number of heat exchanger units. 
The third step consists in finding the best heat exchanger network configuration, by means of minimum investment area network. The Multi-period Minimum Investment Network formulation of Floudas and Grossmann (1987) is tuned in order to include the design of utilities heat exchangers not only at top and bottom of the heat cascade, but also for those utilities that are across the heat cascade. Additionally, regarding the heat exchanger network configuration, parallel, series and hybrid configuration of utility exchangers are considered in the superstructure. Moreover, the modelling of heat exchangers bypass is avoided by considering Amax as design variables, additionally, despite some literature cases 13,23, the optimization of HRATs of streams for all periods will guarantee to maximize the utilization of the available area, if necessary.

The above-described sequential framework is capable of performing the combined multi-period synthesis and design of both utility systems and heat exchanger networks with reasonable computational times, of the order of a few seconds for industrial scale problems (e.g., 20 streams and 5 periods). In addition, the proposed approach can be used to generate a sequence of improving configurations and designs by integrating the proposed sequential framework with the recently proposed derivative - free black-box optimization algorithm Martelli and Amaldi (2014). In particular, PGS -COM can be used to improve the selection and design of the utility systems and heat exchanger network by optimizing the utility activation, size and design (temperature levels), HRATs of each stream at each time period, and the Penalty Levels (PL) associated to process/process and process/utility heat exchanger units. By exploring the space of utility options, HRATS and PLs, PGS-COM Martelli and Amaldi (2014) allows to overcome the major limits of the sequential approach and find solutions with better trade-off between capital and operating costs compared to the classic sequential approach. It is worth noting that the optimization of the HRAT values over the time periods allows to enhance the energy recovery by better exploiting the available heat exchanger area (e.g., at part-loads, heat exchangers are oversized and then lower values of HRAT should be adopted, leading to a decrease of utility consumption/increase of heat recovery), while the optimization of the penalty levels of the matches allows to explore different matching options between hot and cold streams. A graphical representation of the proposed framework is reported in Figure 1

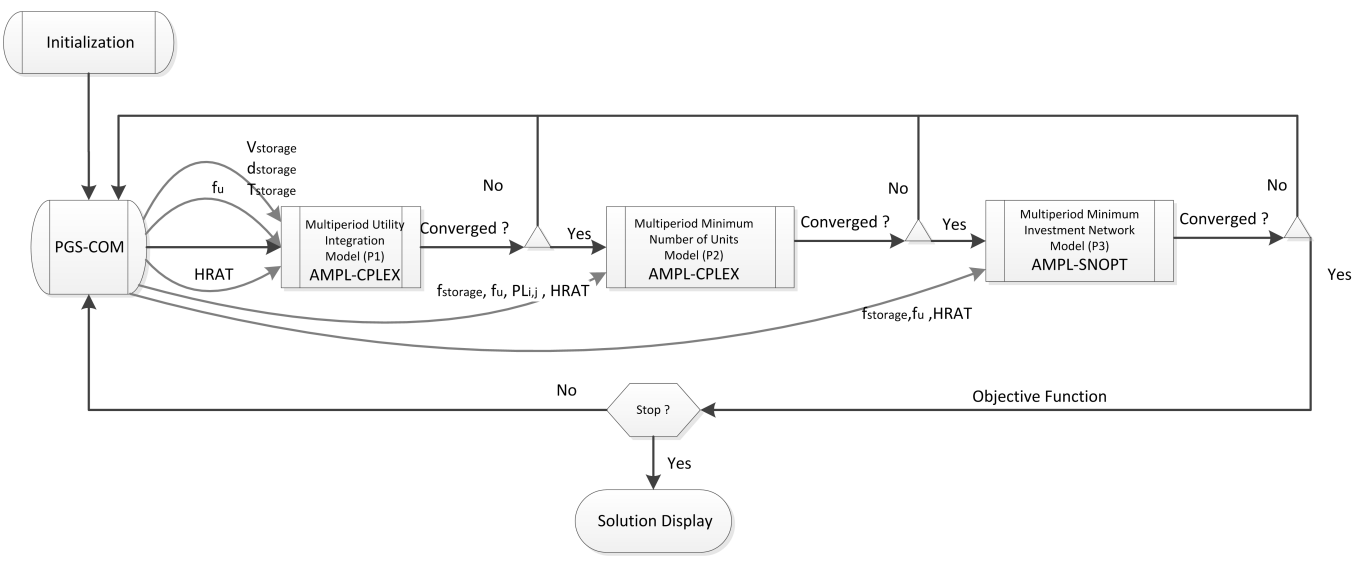

Figure 1: Framework for multi-period utility systems design, schedulign and HENS including thermal storage

\subsection{Thermal storage models}

The superstructure of storage tanks to be included in the problem is automatically generated once the following inputs are specified: number of storage tanks, temperature of each storage tank, stor- 
age volume storage diameter, Heat Transfer Fluid (HTF) density $\rho_{H T F, S T O}$ and HTF heat capacity $c_{p, H T F, S T O}$. In order to account heat losses the following strategy is used to guarantee that the energy balance is satisfied:

- The heat losses are calculated for each storage tank as a function of the storage tank heat transfer coefficient (expressed in $\frac{k W}{m^{2} K}$ ), the storage diameter and the period dependent mass content of the storage;

- Top and bottom storage losses are accounted by considering as first approximation a factor $k_{S}$ that multiplies the storage wall losses;

- For all storages minus the hottest one it is considered that non-isothermal mixing is relized by mixing in the considered storage $S$ the mass-flow derived from $S+1$ at temperature $T_{S+1}$ that allows satisfying the equivalent heat losses. This allows to save the amount of heat exchanger area that would be required to balance losses;

- For the hottest storage tank it is assumed that its heat losses are instantly balanced by recirculating the equivalent mass losses with the neighbor colder storage $S-1$. The discharge of the storage $S-1$ is by consequence calculated assuming one contribution for the heat losses of the upper hottest storage;

It should be noted that this approach would result to a non-lienar model if the temperature of each storage, as well as storage volume and diameter would not be set as inputs. Furthermore, all the input parameters and choices can be in principle optimized using PGS-COM. In this paper only the main storage design variables are optimized (storage volume, diameter and temperature of the considered storages).

\section{Test Case and Results}

In order to test the effectiveness of the proposed framework, the literature example reported in Floudas and Grossmann (1987) is taken as a reference. The example considers two process hot and two process cold streams, as well as one hot and one cold utilities. By considering the proposed example the goal is to highlight the improvements that can be obtained from optimizing at the same time utility size, penalty levels, HRATs. Additionally, design variables related to thermal storages, are included at upper level and optimized using PGS-COM. Consequently, the variables that are optimized by PGS-COM are:

- the HRATs of all involved stream and periods (18 variables)

- the penalty levels $P L_{i, j}$ associated to process/process, process/utility and process/storage streams (15 variables)

- the utility size (2 variables)

- the storage volume (2 variables)

- the temperature of the two storages (2 variables)

- the storage diameters ( 2 variables) The total number of variables that are optimized by PGS-COM is thus 41 . The number of objective function evaluations set as stopping criteria is fixed to 12000 and the number of PGS-COM particles to 100 . The objective function which is minimized by PGS-COM accounts for operating costs, annualized investment costs related to the heat exchanger network. The objective function considers the Total Yearly Costs (TYC) or the plant, according to Equation 1.

$$
T Y C=\left[\sum_{u=1}^{n_{u}} C_{o p, f i x, u} y_{u}+\sum_{t=1}^{\text {Times }}\left(\sum_{u=1}^{n_{u}}\left(C_{o p, v a r, u}^{t} f_{u}^{t}\right)\right) t_{p}\right]++\left[\left(\frac{i(1+i)^{n}}{(1+i)^{n}-1}\right) \sum_{e x \in U} c_{e x} A_{e x}^{\beta_{e x}}\right]
$$




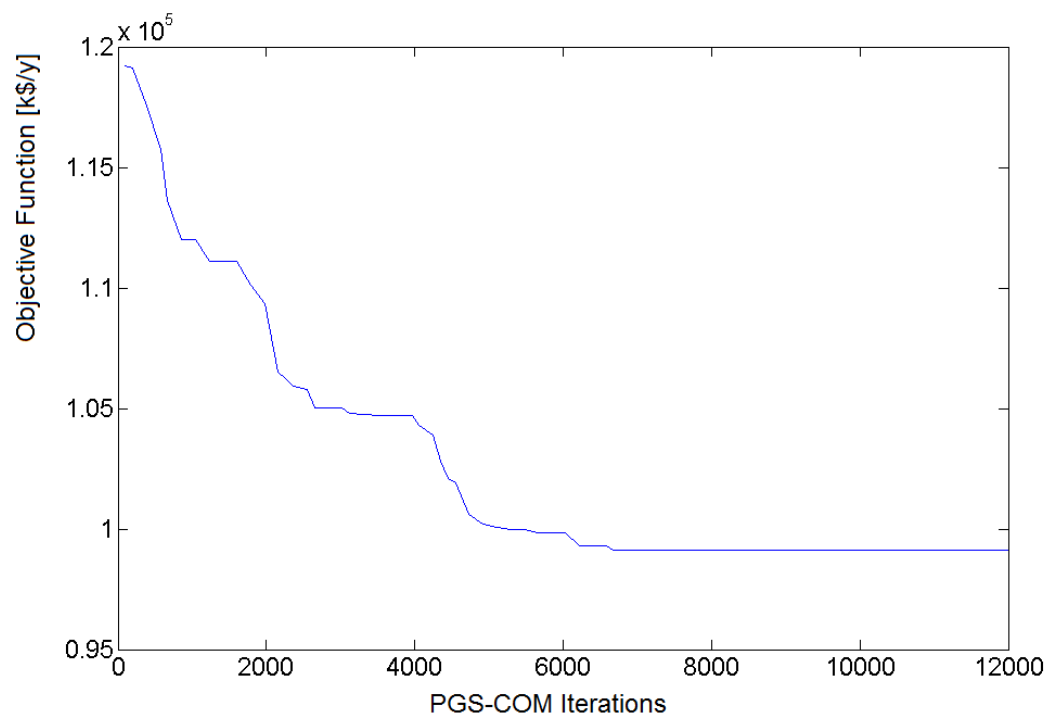

Figure 2: Convergence of PGS-COM for the considered problem

The first term in square brackets is the operating costs considered in (P1) where $C_{o p, f i x, u}$ indicates the fixed operating costs related to the purchase of utility $u, y_{u}^{t}$ denotes the utility selection, $C_{o p, v a r, u}^{t}$ denotes the load and period dependent operating cost of utility $u, f_{u}^{t}$ denotes the load utility at period $t, t_{p}$ denotes the period duration ( 8 hours for each period are considered in our test case). The second term corresponds to the annualized TAC. An actualization factor is required in order to have the discounted price of the HEA, where $i$ is the interest rate of return (fixed to 10\%), $n$ is the lifetime of the exchanger (fixed to 40 years). The investment cost related to a heat exchanger is computed considering a reference area cost coefficient, $c_{e x}$ (set to $4333 \frac{\$}{m^{2}}$ as in the reference test case), and a scaling cost coefficient $\beta_{e x}$ (set to 0.6 in this work). Each objective function evaluation takes on average 1 seconds.

The PGS-COM convergence is reported for the considered iterations in Figure 2 The solution found is compared with the equivalent solution determined by applying the classical sequential framework Floudas and Grossmann (1987). The results in terms of Total Yearly Costs (TYC), computed considering Yearly Operating Costs (OC), Investment Costs (IC) (in particular Discounted Investment Cost (DIC)) of the heat exchanger network are reported in Table 1. Compared

Table 1: Comparison of objective functions between literature work and this work

\begin{tabular}{|c|l|l|l|l|}
\hline & $\boldsymbol{T Y C}[\boldsymbol{k} \mathbf{\$} / \boldsymbol{y}]$ & $\boldsymbol{O C}[\boldsymbol{k} \mathbf{\$} \boldsymbol{y}]$ & $\boldsymbol{I C}[\boldsymbol{k} \boldsymbol{\$}]$ & $\boldsymbol{D I C}[\boldsymbol{k} \mathbf{\$} \boldsymbol{y}]$ \\
\hline Floudas and Grossmann (1986) & 163.92 & 136.99 & 269.38 & 26.93 \\
\hline This framework & 99.04 & 51.019 & 480.33 & 48.03 \\
\hline
\end{tabular}

to the results of the original example, the integration of two thermal storages at different temperatures determine an higher number of exchangers (10 compared to 7 of the original work) with a high investment area cost. On the contrary the benefits in terms of operating costs allows reducing the TYC. With respect to the main assumptions made, considering the three periods cyclic with a pariod duration of 8 hours per each period, the Yearly Total Costs results to be $99.04 k \$$ overy, corresponding to an improvement of $39.5 \%$. The proposed approach can be extended to large-scale problems, the main limitation is the number of variables that can be handled at upper level. The matches, the heat exchanged in each period and the heat exchangers area are reported in Table 2 
Table 2: Optimal Matches and Heat Exchanger Area

\begin{tabular}{|lllll|}
\hline Match & $\begin{array}{l}\text { Period 1 } \\
\text { Heat Load }[\mathbf{k W}]\end{array}$ & $\begin{array}{l}\text { Period 2 } \\
\text { Heat Load }[\mathbf{k W}]\end{array}$ & $\begin{array}{l}\text { Period 3 } \\
\text { Heat Load }[\mathbf{k W}]\end{array}$ & $\begin{array}{l}\text { Heat Exchanger } \\
\text { Area }\left[\mathrm{m}^{2}\right]\end{array}$ \\
\hline HU-C2 & 263.66 & 431.61 & 0 & 18.68 \\
\hline H1-CU & 308.24 & 0 & 551.55 & 28.69 \\
\hline H2-C2 -1 & 122.07 & 768.04 & 219.608 & 28.35 \\
\hline H2-C1 & 676.65 & 0 & 207.26 & 20.71 \\
\hline H2-C2-2 & 859.73 & 0 & 0 & 92.56 \\
\hline H2-STCU & 0 & 0 & 1231.59 & 166.87 \\
\hline H1-C2 & 1214.54 & 766.49 & 1020.39 & 127.57 \\
\hline STHU-C1 & 0 & 676.65 & 0 & 79.99 \\
\hline STHU-C2 & 0 & 493.8 & 0 & 67.33 \\
\hline STHU-CU & 0 & 61.07 & 0 & 4.84 \\
\hline
\end{tabular}

\section{Conclusions}

In this paper a framework which combines the multi-period utility sizing, scheduling and the multi-period sequential synthesis of heat exchanger networks was proposed. The derivative-free, hybrid algorithm PGS-COM has been coupled with the sequential approach for multi-period HENS. At the upper level PGS-COM optimizes the 41 continuous variables (HRATs, Penalty Levels, Utility Size and Storage Design Variables), while at the lower level the sequential framework allows to optimize the design and the multiperiod operation of both utility systems and HEN.. An example was proposed in order to compare the here proposed framework with a literature case. Although it was necessary to limit the number of optimization variables at the upper level, results indicated that optimizing penalty, utility sizes and design variables of storages yields to a considerable improvement $(39.5 \%)$ of the total annual cost with respect to the original example.The main limitation of the proposed approach is the number of variables that need to be optimized at master level. On the other hand, a great advantage of the approach is the possibility of significatnyl reducing the computational time by exploiting the parallel computing capability of PGS-COM.

\section{References}

Chen, C.-L., Ciou, Y.-J., 2008. Design and optimization of indirect energy storage systems for batch process plants. Industrial \& Engineering Chemistry Research 47, 4817-4829.

Christidis, A., Koch, C., Pottel, L., Tsatsaronis, G., 2012. The contribution of heat storage to the profitable operation of combined heat and power plants in liberalized electricity markets. Energy 41, $75-82$.

Floudas, C. A., Grossmann, I. E., 1986. Synthesis of flexible heat exchanger networks for multiperiod operation. Computers \& chemical engineering 10, 153-168.

Floudas, C. A., Grossmann, I. E., 1987. Automatic generation of multiperiod heat exchanger network configurations. Computers \& chemical engineering 11, 123-142.

Grossmann, I. E., Santibanez, J., 1980. Applications of mixed-integer linear programming in process synthesis. Computers \& Chemical Engineering 4, 205-214.

Marechal, F., Kalitventzeff, B., 2003. Targeting the integration of multi-period utility systems for site scale process integration. Applied thermal engineering 23, 176-1784.

Martelli, E., Amaldi, E., 2014. Pgs-com: A hybrid method for constrained non-smooth black-box optimization problems: Brief review, novel algorithm and comparative evaluation. Computers \& Chemical Engineering 63, 108-139. 